\title{
Theory of Error in Geochemical Data
}

GEOLOGICAL SURVEY PROFESSIONAL PAPER 574-A

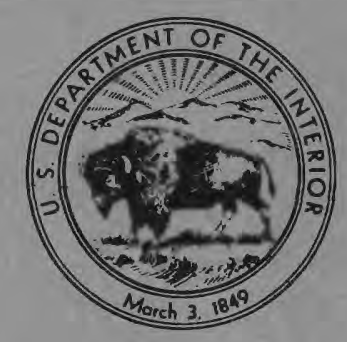




\section{Theory of Error in \\ Geochemical Data}

By A. T. MIESCH

STATISTICAL STUDIES IN FIELD GEOCHEMISTRY

GEOLOGICAL SURVEY PROFESSIONAL PAPER 574-A

$A$ classification of geochemical errors

and discussion of their effects in

data interpretation

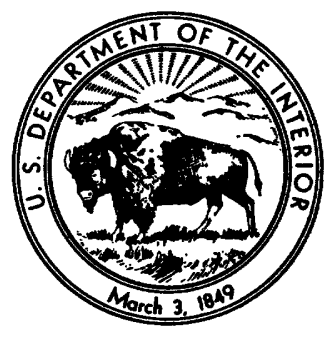




\section{UNITED STATES DEPARTMENT OF THE INTERIOR \\ WALTER J. HIGKEL, Secretary \\ GEOLOGICAL SURVEY \\ William T. Pecora, Director}




\section{CONTENTS}

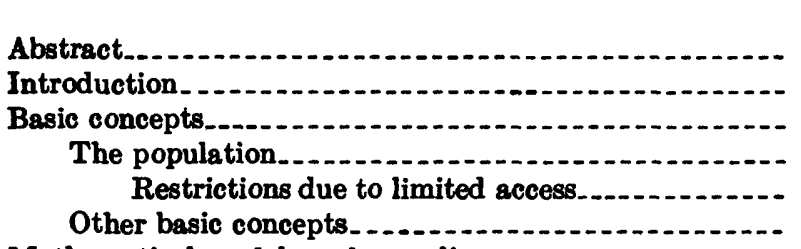

Mathematical models and sampling error.

\begin{tabular}{r|} 
Page \\
A1 \\
1 \\
2 \\
2 \\
2 \\
4 \\
5
\end{tabular}

Special models for geochemical sampling

Analytical error . . . . . . . . . . . . . . .

Effects of error types in statistical analysis............ Analysis of variance..........

Trend analysis. . . .

Summary -.........

Literature cited
Page

A9

10

13

14

14

15

16

\section{ILLUSTRATIONS}

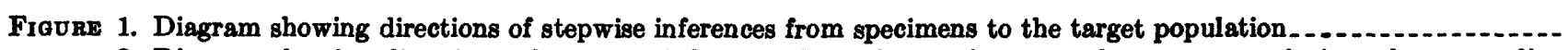

2. Diagram showing directions of stepwise inferences from the specimens to the target population where sampling localities comprise subpopulations.

3. Graphs of analytical determinations on prepared standards of detergent in water

Parge

A3 


\title{
STATISTICAL STUDIES IN FIELD GEOCHEMISTRY
}

\section{THEORY OF ERROR IN GEOCHEMICAL DATA}

\author{
By A. T. Miesch
}

\begin{abstract}
All geochemical data accumulated in field studies directed at the determination of spatial variation in rock bodies contain error due to both sampling and analysis. Sampling error associated with a rock specimen is the compositional difference between the specimen and the part of the rock body that the specimen is intended to represent. Analytical error is the difference between the analysis and the concentration of the constituent in the total specimen. Error that is unbiased and of about equal variance from one sampling locality to another may not greatly hinder attempts at data interpretation; additional data will always serve to reduce the error effects. However, either sampling or analytical error may contain an overall bias (when the mean error does not tend toward zero), a variable bias (when bias is variable from one sampling locality to another), or variable precision (when the variance of the error differs from one locality to another). Errors of these types may signifieantly affect interpretation, and their relative importance is about the same whether the methods of interpretation are based on formal statistical procedures or on intuitive judgment.

Some types of error can commonly be avoided or controlled by taking suitable precautions in the field and laboratory. But where errors result from conditions of the rock body and its outerop or from an inherent shortcoming of an analytical technique that must be used, they are unavoidable. Detrimental effects of some error types may be overcome by suitable data transformation prior to interpretation. Other types of error, if of sufficient magnitude, may invalidate any attempt at interpretation.
\end{abstract}

\section{INTRODUCTION}

Geochemical field studies are highly varied in scope and objectives, but they are most commonly designed to describe and interpret the compositional variation within bodies of rock, soil, or alluvium, or the compositional variation among different rock bodies. The distinction between these two types of problems is not necessary to a discussion of error, and here we need consider only the variation within a single rock body. Depending on how it is defined, this rock body may or may not contain sharp contacts or discontinuities that would allow its division into multiple rock bodies.

Any geochemical field study of this kind involves the problems of obtaining specimens to represent larger bodies of rock and of obtaining analyses to represent the specimens. Thus, two main kinds of error-that due to sampling and that due to analysis-are always present, even though the importance of each may vary greatly from one study to another. Both the type and the magnitude of error should be considered; some types of error need not seriously affect data interpretation even if they are large. Other types can never be overcome and, if of sufficient magnitude, have serious effects on data interpretation.

A classification of types of error important in geochemical prospecting was described briefly in a previous paper (Miesch, 1964). The same classification as it applies to a broader class of geochemical field problems is presented here, along with some basic concepts that may be essential to understanding the nature of error. Geologists have always employed most of the concepts, but some geologists express them in terms of a rigid statistical framework within which experiments in field geochemistry are carried out, whereas other geologists view the statistical framework informally and conduct their experiments according to intuitive judgment. Actually, the concepts employed by the two groups are virtually the same. Moreover, there are few requirements demanded of data, and of data errors, for rigid statistical analysis that are not demanded otherwise, and it can be shown that sampling and analytical errors similarly affect statistical and nonstatistical interpretations of the data. The concepts and mathematical frameworks used in statistical experiments, and the requirements and assumptions regarding data errors, are not so different from those most geologists have been employing for many years without formal statistical analysis.

The principal theme of this report is in support of the notions presented in the preceding paragraph. The report aims to show that adoption of rigid experimental design techniques in field geochemistry does not necessarily require radical departure from methods and concepts now being used, but, instead, requires mainly a greater awareness of things we already know. Statistical principles are commonly stated for 
the general case, and their application to individual problems is not always obvious. It is hoped that this discussion will be of some help in interpreting a few basic statistical principles in terms of the particular situations in field geochemistry. To avoid making a subject that is already seemingly complex even more so, the geologic and analytical problems used as examples will be kept simple, perhaps even trivial. The examples, however, will be ones that are familiar to nearly every field geochemist or petrologist.

Geochemical errors in general are departures of determined values from true or expected values or from values one is attempting to estimate; but such a departure does not necessarily imply that a mistake has been made in sampling or analysis. According to definition (Webster's Seventh New Collegiate Dictionary, 1963, p. 282), the term "error" "*** may suggest an inaccuracy where accuracy is impossible." Error may result from mistakes or blunders in rela tively rare cases. Thus, the degree or error in a particular geochemical study may be controlled by the nature of the study itself, and not necessarily by the care with which the study is conducted. Two geochemical studies that are identical in objectives and are conducted with identical degree of precaution in sampling and analysis may produce different types and magnitudes of error, depending on the natures of the two areas or rock bodies involved or on the methods of analysis used. In studies directed at rock bodies that are highly variable in composition, more sampling error must be expected than would be met in studies of relatively homogeneous rocks. Where rapid lowcost analytical methods are used, the errors from analysis are commonly greater than where more elaborate methods are used, but the high precision of the elaborate methods may be unnecessary, and of little advantage, in studies where sampling error is large.

Acknowledgments.-I am grateful to Dr. W. C. Krumbein, Department of Geology, Northwestern University, and to Dr. R. F. Link, Department of Mathematics, Princeton University, for valuable criticism of an early draft of the manuscript.

\section{BASIC CONCEPTS}

\section{THE POPULATION}

Geochemical sampling is normally directed at rock bodies, or parts of them, whether they are granitic plutons, selected stratified layers, covers of soil or alluvium, or whatever is of geologic interest. The boundaries of some rock bodies are distinct and well defined. The boundaries of others are arbitrary and may or may not be well defined. Nevertheless, the rock body is a single unit and cannot, while considered in this sense, be regarded as a geologic population; moreover, a rock body in this sense has a bulk composition but has no mean composition or compositional variance until it is conceptually divided into parts. In studying a rock body it is convenient for the geologist to collect specimens or samples, and the population is frequently regarded as all the potential specimens that the rock body contains. The fact that some potential specimens cannot be obtained has led to the concepts of target and sampled populations introduced by Cochran, Mosteller, and Tukey (1954) and used by Krumbein (1960, p. 351).

Characteristics of the population of specimens, such as the variance, may largely depend on specimen size. In a study of uranium ores from the Colorado Plateau, Shoemaker, Miesch, Newman, and Riley (1959) used samples split from crushed and homogenized ore shipments aggregating at least several tons. The compositional variation among these samples was less than might have been found in samples cut from only a few pounds. If the samples were so small that each included only a few mineral grains, the amount and nature of the variation from one sample to another, again, would be entirely different. Laffitte (1957, p. 101-105) discussed efficiency of sample size in relation to size of constituent mineral grains.

The geologic population in geochemical sampling, then, is determined by the geologist when he decides on the target of his investigation. Commonly the population consists of all possible specimens of about a few pounds' size that occur within a rock body. Because most rock bodies under study are large, and because possible specimens can overlap, the population is, for all practical purposes, infinite in size.

\section{RESTRICTIONS DUE TO LIMTTED ACCESS}

Although the geologic population of interest in most geochemical field studies comprises all possible specimens occurring within a rock body, sampling is generally limited to parts of the rock body that are exposed at the surface. In some work, mostly that bearing on resource appraisal, deep drilling can be conducted in such a way that the entire rock body is theoretically accessible for sampling; in other work, shallow drilling or trenching may furnish samples from within a few feet of the surface. Except where drilling or trenching makes the entire rock body accessible for sampling, there may be a large difference between the composition of the target population (the population of interest) and the sampled population (the population that can be sampled). The difference is due to both original compositional variation in the rock body and secondary variation caused by weathering and other related processes. 
Sampling limitations frequently necessitate that one or a series of indirect inferences about the target population be made from the collected specimens. The inferences may proceed in stepwise fashion, as illustrated in figure 1. The correctness of the inferences may partly depend on the degree to which the sampled population corresponds to the target population; the sampled population, in turn, may be determined by the facilities available for sampling.

If facilities such as deep-drilling equipment are available, making the entire rock body accessible for sampling, the inference from the specimens to the target population may be direct and straightforward. Objective schemes of drilling and of sampling the drill core or cuttings would be especially helpful in validating the inference.

Where shallow-drilling devices or trenching equipment is available, the sampled population may consist of all possible specimens from within a few feet of the surface ( $A$, fig. 1$)$. Inferences from the specimens to this sampled population may be direct and straightforward, but the additional inference from the sampled population to the target population is more difficult. The validity of this second inference depends on the nature of the rock body being studied, especially on such factors as compositional zoning and depth of weathering.

If the sampling devices are used only to obtain specimens from the outcropping parts of the rock body (such devices are necessary, for example, in sampling a smooth face of dolomite or ganite that cannot be sampled satisfactorily with a hammer and chisel; see Baird and others, 1964, p. 258), an additional indirect inference may be required to interpret the nature of the rock body from the nature of the specimens ( $B$, fig. 1$)$. One must infer that the outcropping part of the rock body adequately represents the part of the rock body near the surface, and that this part, in turn, adequately represents the rock body as a whole.

In most geochemical field problems the only available sampling devices are a hammer and chisel or similar tools. (Most rock specimens are collected by means of only a geologic pick and hammer. However, the portion of an outcrop that can be sampled with an ordinary pick and hammer is commonly greatly limited in comparison with that which can be sampled using a 2-pound sledge hammer and a heavy steel cold chisel. Thus, bias in sampling may be significantly reduced by using a chisel for sampling many types of rock.) Consequently, depending on the type of rock being studied, not all parts of the outcrop can be sampled. Specimens are generally taken only where the rock protrudes, as along bedding planes or fractures. This is especially true where the rock is hard and dense, as are many quartzites, dolomites, and granites. Where a hammer and chisel are used as sampling devices, the sampled population consists only of specimens that can be collected with this equipment ( $C$, fig. 1$)$, and the inferences to be made about the target population from the collected specimens, with few exceptions, are subject to severe limitations.

These few examples illustrate the kinds of problems met in inferring the composition and compositional variation of an entire rock body from specimens collected with limited sampling access, but the problems are similar to those commonly encountered. The difficulty of inferring from the sampled populations ( $A, B$, and $C$, fig. 1) to the target population, as defined in figure 1, may discourage one from such attempts.

In some specialized geochemical problems the target population is not the potential specimens from the entire rock body but only those near or at the surface. This is true, for example, in studies directed at environmental health problems, because it is primarily the

Target population:

Sampled populations:

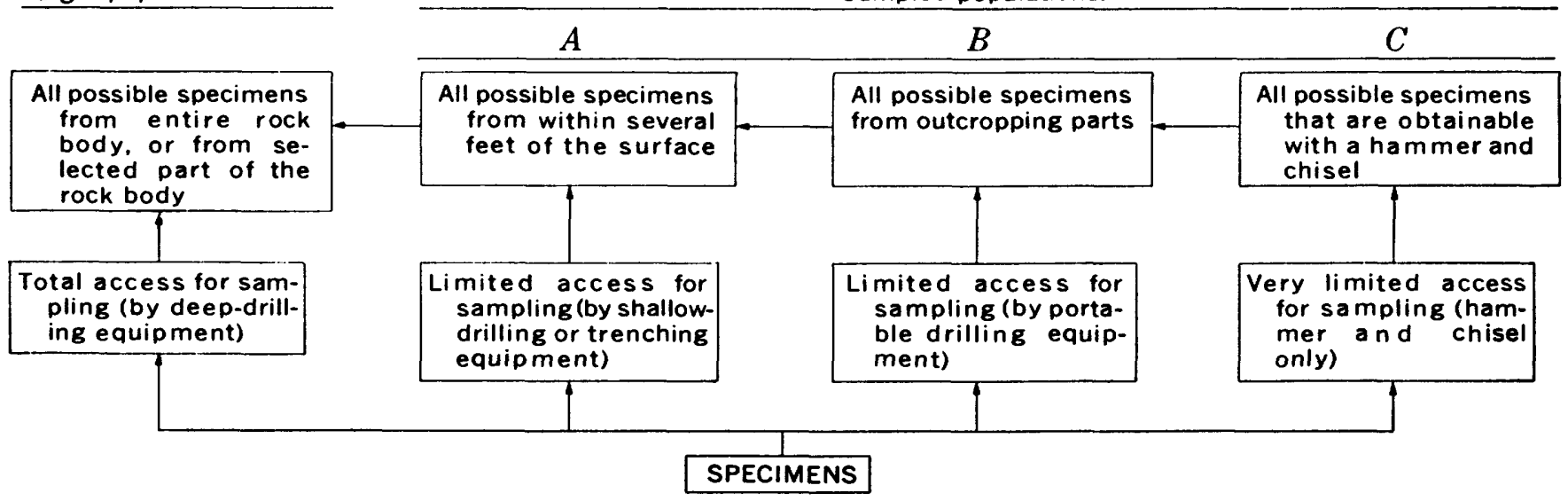

Fiaure 1.-Directions of stepwise inferences from specimens to the target population. 
near-surface part of the rock body that affects this environment. This may also be true in geochemical exploration problems where the target population comprises only the upper few inches of soil or alluvium; here the target population corresponds to the sampled populations designated by $A$ or $B$ in figure 1 , and the inference from the specimens to the target population may be direct and straightforward.

In other geochemical problems the inference from sampled to target population may be so difficult that the geologist confines his assessments of geochemical properties to the sampled population, labeling any further inference as speculation only.

Where the target population corresponds to the part of the rock body that is accessible for sampling, or where inferences are confined to the sampled population, sampling bias can generally be eliminated. Specimens, in these studies, may always be collected by some objective operational procedure (Krumbein, 1960, p. 351).

In many geochemical field problems it is convenient to employ sampling localities, each locality being defined as some restricted part of the total rock body. Each part of the rock body within a sampling locality, then, contains a target subpopulation, and the portion of the target subpopulation that is accessible for sampling is a sampled subpopulation. Further discussion of sampling localities and subpopulations is given in a following section.

Krumbein (1960) presented a comprehensive discussion of the concepts of target and sampled populations as they may be applied in geology.

\section{OTHER BASIC CONCHPTS}

Other concepts basic to the theory of error in geochemistry are accuracy, bias, precision, and variance. A few additional remarks concerning the experimental nature of geochemical field investigations may also be in order.

Accuracy, as used in this discussion, implies close agreement between the average of a large number of estimates and the correct value. The difference between the average of a large number of estimates and the correct value is called bias. Differences between the average of a small number of estimates and the correct value may or may not reflect bias; if the difference tends to diminish toward zero as the number of estimates is increased, bias is absent and the method of estimation is said to be accurate. In practice, of course, the correct value is never known and is taken as a value derived by some technique considered inherently more likely to be correct than the technique used for arriving at the estimate.
In theory of statistical estimation, usage of the term "accuracy" differs somewhat from that given in the preceding paragraph, and the term "consistency" is frequently used in much the same manner that "accuracy" is used here. The distinction is necessary only in discussing relative merits of various formulas used in estimation. As we are concerned here only with errors arising from sampling and analysis, the term "consistency" is of little use. The estimation formulas referred to are both accurate (unbiased) and consistent, even though they may not be the most efficient in certain problems. The terms "accuracy," "consistency," and "efficiency" in statistical estimation are discussed in numerous texts, including those by Ostle (1963, p. 87) and Bennett and Franklin (1954, p. 134-136, 209).

Precision implies reproducibility, or close agreement among replicate estimates, and is independent of accuracy. Methods of estimation may be both precise and accurate, neither precise nor accurate, precise but not accurate, or accurate but not precise (Ostle, 1963, p. 105).

The accuracy and precision of individual estimates, whether they are chemical determinations, statistical averages, or other types, depend on the accuracy and precision of the method by which they are derived.

Imprecision, or the lack of perfect reproducibility in measurements, is the type of error that statistical methods are designed to overcome and is the least serious type of error which can occur. Moreover, imprecision can always be effectively overcome by the collection of more data (by additional sampling or by replicate analyses of specimens).

Variance is a statistical device for describing the degree of variation among a set of variables or measurements. Considering $X_{j}$ as the $j$ th measurement in a group of $n(1 \leq j \leq n)$, the variance of $X$ is estimated by:

$$
s_{X}^{2}=\frac{1}{n-1} \sum_{j=1}^{n}\left(X_{j}-\bar{x}\right)^{2},
$$

where $\bar{x}$ is the arithmetic mean of the $n$ values. Among the reasons for the widespread utility of variance is its property of additivity (Tippett, 1952, p. 167). When two or more sets of independent variables are added, the sum of their variances, each based on $n$ measurements, is equal to the variance of the $n$ sums. This can be illustrated with a small group of numbers if the numbers are adjusted to be perfectly independent, by eliminating spurious effects, as these effects tend to be eliminated in large sets of data. Using the columns of numbers below, suppose that duplicate analyses $(j=1$ and $j=2)$ have been made of two specimens $(i=1$ and $i=2)$ so that four analytical values, $X_{i j}$, are available. Suppose 
also that each value is determined by the sum of an unknown correct value for the specimen, $T_{i}$, and an unknown analytical error, $\alpha_{i j}$.

\begin{tabular}{|c|c|c|c|c|c|c|}
\hline F & $j$ & $X_{i j}$ & $=$ & $T_{i}$ & + & $\alpha_{i j}$ \\
\hline & 1 & 15 & & 12 & & +3 \\
\hline & 2 & 5 & & 12 & & -7 \\
\hline & 1 & 15 & & 14 & & +1 \\
\hline & ? & 0 & & 14 & & \\
\hline
\end{tabular}

Adjustment of the numbers in this example consisted of fixing the mean of $\alpha_{11}$ and $\alpha_{12}(+3$ and -7$)$ to equal the mean of $\alpha_{21}$ and $\alpha_{22}(+1$ and -5$)$, so that the correlation between $T$ and $\alpha$ is exactly zero. The variances of $X_{i j}, T_{i}$, and $\alpha_{i j}$ are $24,1 \frac{1}{3}$, and $22 \%$, respectively, as derived from equation 1 . The variance of $T_{t}$ is the between-sample variance component, and that of $\alpha_{i j}$ is the within-sample, or error, variance. Wherever these two quantities in real data are additive, analysis of variance procedures can be used to estimate each of them. They will not be additive unless the errors are independent-a property insured in this artificial example by fixing the mean errors for each specimen to be equal. In actual data sets the mean errors will not be precisely equal, but satisfactory estimates of the variance components can be obtained if the mean errors tend to be equal as the number of values in the experiment is increased.

Because of the additive character of the variance, data from suitably designed experiments can be used to apportion the total variability among geologic or other factors considered in an experiment through analysis of variance techniques. These techniques, as well as other techniques of statistical analysis, are based on formal mathematical models discussed in following sections.

Geochemical field investigations may be regarded as "experiments," even though geologists rarely use this term in oral or written discussions. Use of the term, however, helps to clarify the fact that each geochemical field investigation is a trial that is subject to repetition and confirmation. If the experiment is conducted according to some definite operational procedure, it can be replicated and confirmed; differences among replicate experiments can be used to measure the precision (reproducibility) of the experimental results.

\section{MATHEMATICAL MODELS AND SAMPLING ERROR}

Mathematical models are used either formally or informally in all geochemical sampling. Informal models are used when the geologist is attempting to estimate amounts or differences but does not formalize the problem in a mathematical equation. The nature of the model depends on the purpose of the specific study. The purpose may be, for example, to estimate the composition of the total rock body, to estimate its variation in composition, or, commonly, to determine the spatial variation with respect to geologic factors such as sedimentary source areas, the margins of igneous plutons, ore deposits, and structural features. In this section some simple models for geochemical field problems will be developed and used to define sampling error. It is assumed here that no analytical error is present. In the following section the basic model will be extended to account for variation resulting from this source.

If a rock body were perfectly homogeneous on a specimen scale, so that every potential specimen had the same composition, the model might be expressed as:

$$
X_{j}=\mu \text {, }
$$

where $X_{j}$ is the concentration of some constituent in the $j$ th specimen, and $\mu$ is the mean concentration for all specimens in the population being studied. That is, each specimen would have the same compositionor the composition of each specimen would be equal to the mean composition of all specimens. Clearly, sampling error would be impossible if such an idealized rock body existed.

A more realistic model might be written as:

$$
X_{j}=\mu+v_{j} .
$$

Here each specimen is regarded as having a composition determined by $\mu$, the arithmetic mean for all specimens in the population, plus a positive or negative deviation, $v_{j}$, from the mean. The purpose of much geochemical sampling is to estimate $\mu$, to estimate the expected magnitude, or range, of $v_{j}$, or to examine the magnitude of $v_{f}$ with respect to geologic or spatial factors.

If the geologist is actually interested in the variation among potential specimens over the entire rock body, and no one part of the rock body is of any greater interest than another part, it seems appropriate to collect specimens randomly, or at about even intervals over the entire rock body, or over the part that is accessible for sampling. Sampling need not be either random or on a grid, but the sampled portions of the rock body should not be purposely clustered.

If the rock body is relatively homogeneous on a local scale, the collection of a small number of specimens broadly distributed over the rock body may be effective. However, if the rock is locally heterogeneous, the local variation will complicate detection of regional differences. The effects of the local variation may be at least partly overcome by employing sampling localities-each locality defined as a part of the rock body larger than a single specimen-and collecting a number of samples at each locality. The local 
variation is smoothed (or "averaged out") in this manner.

Employing sampling localities requires a revised approach to the field problem. The objective is to determine not the variation among specimens but the variation among parts of the rock body larger than a specimen. According to terminology of Krumbein and Slack (1956, p. 744), these larger parts may be referred to as "master sampling units," or according to more common terminology, as "major sampling localities." Each specimen, or group of specimens, collected within a major sampling locality, then, is intended to represent the locality-a target subpopulation. A locality may be defined as a point on a sampling grid, a cell within a grid, an outcrop, or a stream valley, or in any manner convenient and meaningful for the particular field problem. The purpose is to determine the amount and nature of variation among localities (among locality means); variation among specimens collected within a locality is of no particular interest, except that it determines what may be called the residual variation (or the withinlocality variation) and controls the number of specimens that must be collected in each locality to represent the locality adequately. (See Miesch and Connor, 1964, p. D84.)
When sampling localities are employed as described here, the stepwise inferences from the specimens to the target population (fig. 1) must also be reframed. (See example in fig. 2.) If that part of the rock body that constitutes a locality is completely accessible for sampling, inferences from the specimens to the locality may be direct and straightforward. If it is not completely accessible for sampling, direct inferences may be made about the part that was sampled (the sampled subpopulation), but further inferences concerning the total locality (the target subpopulation) are necessary. Finally, inferences may be drawn about the total rock body - the prime target population-from the nature of the localities. The validity of these inferences depends partly on the spacing of sampling localities, which, in turn, may depend on the purpose of the experiment. These matters, beyond the scope of this paper, occur within the realm of sampling design problems.

Where major sampling localities are employed in a geochemical field investigation, the model in equation 3 is extended to:

$$
X_{i j}=\mu+\beta_{i}+\omega_{i j}
$$

Here $X_{i j}$ is the quantity of some constituent in the $j$ th specimen from the $i$ th locality. The term $\mu$, again, is the grand mean of the constituent of all possible speci-

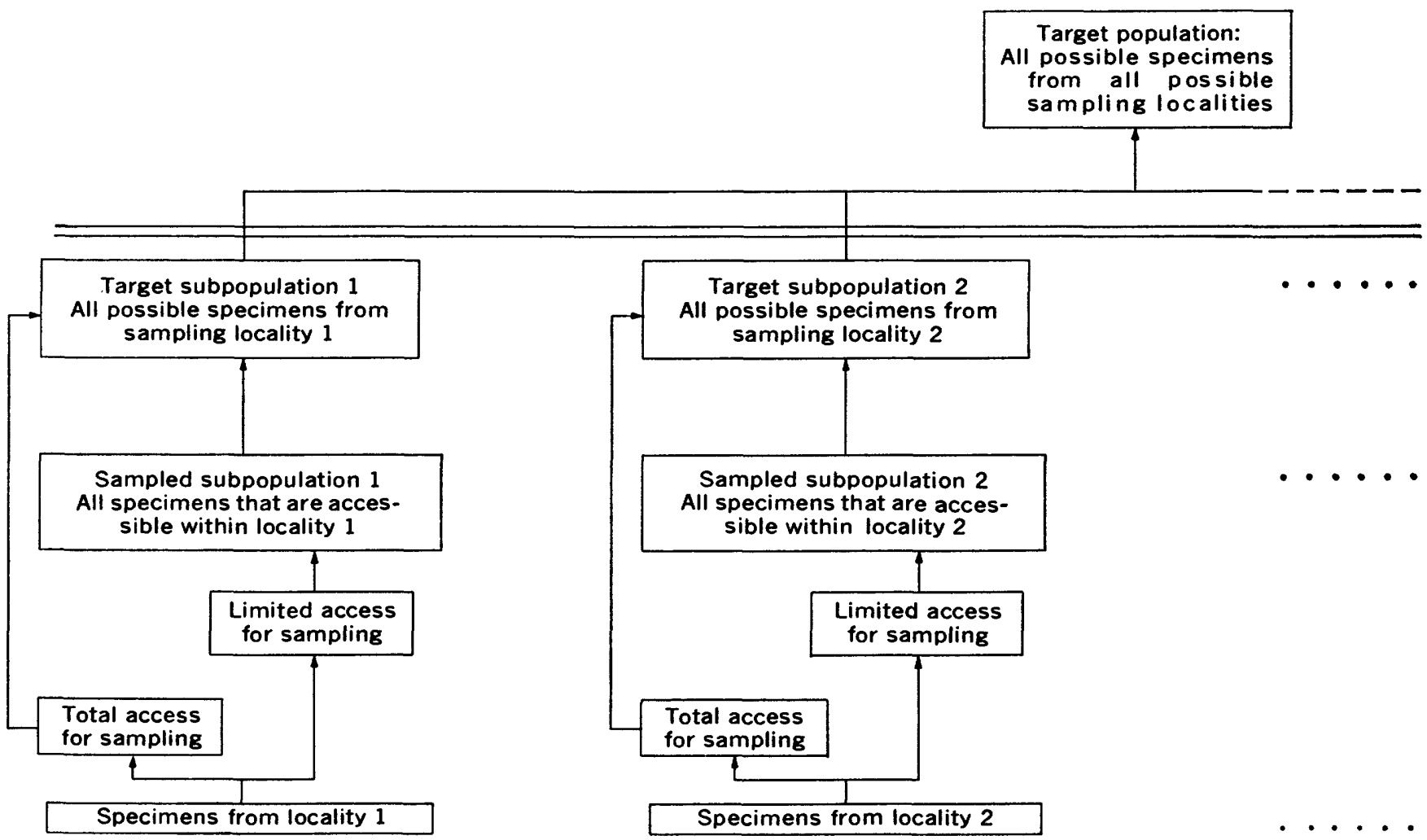

Flavre 2.-Directions of stepwise inferences from the specimens to the target population where sampling localities comprise subpopulations. 
mens in the total rock body. The $v_{j}$ component in equation 3 is now represented by two components, $\beta_{i}$ and $\omega_{i j}$, where $\beta_{i}$ is the deviation of the $i$ th locality mean from the grand mean, $\mu$, and $\omega_{i j}$ is the deviation of the $j$ th sample from the $i$ th locality mean.

The value $\mu+\beta_{i}$ is the true mean concentration of a constituent in the $i$ th locality (the $i$ th subpopulation), and the quantity $\mu+\beta_{i}+\omega_{i j}$ is the true concentration in the $j$ th specimen from the $i$ th locality. The $\omega_{i j}$ component is the difference between the concentration in the specimen $\left(\mu+\beta_{i}+\omega_{i j}\right)$ and the true mean for the locality $\left(\mu+\beta_{i}\right)$.

As the purpose of the $j$ th specimen from the $i$ th locality is to represent the mean of the $i$ th locality $\left(\mu+\beta_{i}\right)$, the component $\omega_{i j}$ may be regarded as the sampling error associated with the specimen, and the mean of all the $\omega_{i j}$ components associated with specimens from the $i$ th locality is the sampling error associated with the locality. Thus, sampling error is caused by local variation within the rock body. Relatively homogeneous rock bodies can be sparsely sampled with little chance of large sampling error, whereas highly variable rock bodies require taking a large number of specimens to represent them adequately.

The $\omega_{i j}$ component associated with the specimen or the mean $\omega_{i j}$ component associated with a sampling locality is never known in practice. Its computation would require prior knowledge of $\mu+\beta_{i}$, the true mean for the locality, and this, in turn, would require complete sampling of the locality and analysis of the specimens (determination of $X_{i j}$ ) by a perfectly accurate and precise technique. Nevertheless, we must make judgments about $\omega_{i}$, prior to any sort of interpretation of geochemical data.

Four aspects of the $\omega_{i j}$ components are discussed next. Three are of particular significance, regardless of whether the interpretation is statistical or intuitive. The fourth is important mainly where the statistical methods used involve certain kinds of probability theory.

Furst aspect

The grand mean of the sampling error components $\left(\omega_{i j}\right)$ over all specimens and localities should tend toward zero as the number of localities, $m$, and of specimens per locality, $n$, is increased. That is,

$$
\frac{1}{m n} \sum_{i=1}^{m} \sum_{j=1}^{n} \omega_{i j} \rightarrow 0, \text { as } m, n \rightarrow \infty \text {. }
$$

If equation 5 is not true, an overall bias is present in the data. Such bias may occur when the total rock body (the target population), or the total sampling locality (the target subpopulation), is not accessible for sampling, or when sampling is not done according to some objective operational procedure (Krumbein,
1960 , p. 351). In the latter instance the selection of samples may involve personal bias on the part of the sampler. Personal bias may be easily avoided, but bias caused by limited accessibility of the rock bodysuch as lack of outcrop-is more difficult to overcome.

For example, parts of the rock body rich or deficient in some constituent may tend to be either covered or to crop out as smooth surfaces that are difficult to sample with a hammer and chisel, as previously discussed. In this circumstance the geologist may resort to another sampling device, such as a portable drill. If drilling or other sampling devices are not practical, and the outcropping parts of the rock body are unlike the total sampling locality, the inference from the sampled subpopulation to the target subpopulation (fig. 2) may be difficult. Nevertheless, this is the common situation, and consideration of possible differences between the sampled and target populations is required in most field studies.

Where the target subpopulation, however defined, is completely accessible for sampling, objective sampling procedures (commonly involving some randomization in sample selection) can reduce or eliminate the possibility of personal bias.

Overall bias in sampling, if of sufficient magnitude, may seriously affect the estimation of means, but it will not hamper attempts to describe variation among sampling localities. Unless the bias differs from one locality to another, as discussed under aspect 2 , the errors in locality means tend to be constant, and estimates of differences among means tend to be correct.

\section{second aspect}

The mean of the sampling-error components should tend toward the same value for each locality as the number of specimens per locality is increased. That is,

$\frac{1}{n_{1}} \sum_{j=1}^{n_{1}} \omega_{1 j} \rightarrow \frac{1}{n_{2}} \sum_{j=1}^{n_{3}} \omega_{2 j} \rightarrow \ldots \frac{1}{n_{i}} \sum_{j=1}^{n_{1}} \omega_{i j}$, as $n_{1}, n_{2}, \ldots n_{i} \rightarrow \infty$,

where $n_{i}$ is the number of specimens from the $i$ th locality. Where equation 6 is not true, variable bias is present. If each term in equation 6 tends toward zero, neither variable bias nor overall bias is present. Variable bias in sampling, or bias that varies from one sampling locality to another, may result from differences in the circumstances under which the sampling is done. This can be caused, for example, when localities are sampled by different individuals having different personal biases, or when the outcrop characteristics vary among localities. The problem of personal bias is easy to resolve, but the problem caused by differences in outcrop characteristics is more difficult. For example, all localities within the rock body may actually have the same total composi- 
tion, but parts rich in, say, calcium carbonate may tend to crop out at one locality and to be covered at another. Even objective sampling of the exposures at each locality, then, may result in maps showing regional variation caused perhaps by climatic differences. Variable bias is often unavoidable, and it is therefore necessary to consider all the factors (even those which are nongeological) that may be important in the interpretation of the experimental results. Variable sampling bias, however, can never be completely overcome in data interpretation, and if it is of sufficient magnitude it may seriously hamper attempts to describe regional variation in the rock body. Variable bias, however caused, may be the most serious type of error that can be present in geochemical data.

\section{Thind aspect}

The variation, or precision, of the sampling error, conveniently expressed as the variance, should tend to be the same for each locality as the number of specimens per locality is increased. That is,

$$
\begin{array}{r}
\frac{1}{n_{1}-1} \sum_{j=1}^{n_{1}}\left(\omega_{1 j}-\bar{\omega}_{1}\right)^{2} \rightarrow \frac{1}{n_{2}-1} \sum_{j=1}^{n_{2}}\left(\omega_{2 j}-\bar{\omega}_{2}\right)^{2} \rightarrow \ldots \\
\rightarrow \frac{1}{n_{i}-1} \sum_{j=1}^{n_{i}}\left(\omega_{i j}-\bar{\omega}_{i}\right)^{2} \text {, as } n_{1}, n_{2}, \ldots n_{i} \rightarrow \infty,
\end{array}
$$

where $\bar{\omega}_{i}$ is the mean sampling error component of the $i$ th locality. Using $s_{\omega_{i}}^{2}$ for the variance of $\omega_{i j}$ at the $i$ th locality, equation 7 may be written as:

$$
s_{\omega_{1}}^{2} \rightarrow s_{\omega_{2}}^{2} \rightarrow \ldots s_{\omega_{i}}^{2} \text {, as } n_{1}, n_{2}, \ldots n_{i} \rightarrow \infty .
$$

If equation 8 is not true, variable precision in sampling is present. The conditions defined by equations 7 and 8 , unlike those defined by equations 5 and 6 , may be tested from the sampling results if analytical errors are sufficiently small to insure that sampling precision, rather than analytical precision, is being observed. The ability to test for the relation in equations 7 and 8 results from the fact that the quantity $\omega_{i j}-\bar{\omega}_{i}$ for the $i$ th locality is equal to $X_{i j}-\bar{x}_{i}$. Therefore, equations 7 and 8 are identical with:

$$
s_{X_{1}}^{2} \rightarrow s_{X_{2}}^{2} \rightarrow \ldots s_{X_{i}}^{2} \text {, as } n_{1}, n_{2}, \ldots n_{i} \rightarrow \infty,
$$

where $s_{\mathbb{X}_{i}}^{2}$ is the variance of a constituent in samples from the $i$ th locality.

If all samples from a particular locality have about the same composition, those locality means estimated from repeated sampling experiments will also be closely similar, or reproducible. If, on the other hand, the samples differ widely in composition, the means from repeated experiments, each involving a small number of samples, will also differ widely. The expected variance of means may be estimated for a single set of samples by use of the statistical relation:

$$
s_{\bar{X}_{i}}^{2}=\frac{s_{X_{i}}^{2}}{n_{i}}
$$

That is, the expected variance of means for the $i$ th locality (the degree to which a locality mean may be expected to vary in repeated experiments) is $1 / n_{i}$ of the variance among the samples from the locality.

If objective sampling is employed at all the sampling localities, the variance among collected specimens is controlled by the nature of the rock body (its local variability) and its outcrop characteristics. Variable precision, then, is caused by variation of these factors from one locality to another. For example, samples from one locality may be widely different in composition because the rock body there is highly variable and completely exposed for sampling, whereas samples from another locality may be virtually identical because the rock body is relatively homogeneous or only a relatively homogeneous part of it is exposed. Variable sampling precision may commonly occur in minor-element data because the variation of minorelement concentrations in rocks tends to be proportional to their mean concentration. Where the means vary widely among localities, the variation, and therefore the sampling precision, may also vary widely.

The significance of deviations from the mean is difficult to evaluate where sampling precision is variable. Locality means are estimated with varying degrees of confidence or reliability, and a minor deviation of one locality mean from the grand mean for the rock body may be geologically significant, whereas a major deviation for another locality mean may result only from sampling error. The relative statistical significance and possible geologic implications of deviations are not easily determined from their magnitude.

Variable precision may be overcome, in some experiments, by using suitable data transformations. For example, if variation within localities tends to be proportional to the locality means, the variation of the logarithms of the concentrations tends to be constant among the localities. Transformation of the data to logarithms, in this circumstance, would be a valid and effective means of overcoming variable precision. Transformations must be used cautiously, however, as complicating factors may thereby be introduced into the experiment. Link, Koch, and Gladfelter (1964, p. 35) referred to the difficulties that had to be overcome by Sichel (1952) and Krige (1960) in estimation problems where the data required logarithmic transformation. 
Variable precision of means among localities can be overcome by collecting different numbers of specimens at the several localities. This is equivalent to adjusting the values of $n_{i}$ in equation 10 so that the value of $s_{\overline{\mathbf{x}}}^{\frac{2}{4}}$ tends to be the same across localities. The adjustment of $n_{i}$ values would have to be made on the basis of preliminary estimates of $s \frac{g}{\bar{x}_{i}}$, obtained from a pilot sampling survey (Miesch and Connor, 1964).

Fourth aspect

The sampling errors (the $\omega_{i j}$ components of equation 4) for any particular sampling locality should tend to be normally distributed; that is, the frequency distribution of $\omega_{i j}$ should approximate the bell-shaped normal, or Gaussian, curve. This requirement, as that in aspect 3 , can be tested by using the observed data, $X_{i j}$, if analytical error is relatively small. The frequency distribution of the data, $X_{i j}$, for the $i$ th locality will have the same form as that of the theoretical component, $\omega_{i j}$.

Frequency distributions that are highly asymmetrical are less easily interpreted than symmetrical distributions, regardless of the method of interpretation; but only in methods based on certain probability theory must the frequency distributions be normal (Gaussian). Most of these methods, however, allow at least a moderate, and commonly a large, departure from normalcy.

\section{SPECIAL MODELS FOR GEOCHEMICAL SAMPLING}

The geochemical sampling model given in equation 4 is applicable to the general case where the objective of the field study is to detect and describe the variation among parts of the rock body larger than a single samplo-in other words, among sampling localities. These localities are commonly about evenly spaced over the rock body, or over the part of the rock body that is of interest, and are referred to as the major sampling localities.

More specialized models have been employed in geochemical sampling by Krumbein and Slack (1956) and by Baird, McIntyre, Welday, and Madlem (1964), and models similar to theirs are currently being used in several geochemical investigations by the U.S. Geological Survey. The purpose of each such investigation is to determine the type of variation present in a rock body and to devise an efficient sampling plan based on the type of variation found. Krumbein and Slack, for example, measured radioactivity in a shale bed associated with a cyclothem deposit over a large part of southwestern Illinois. In the regional phase of their study they collected and grouped samples by source as within mines, selected mines within townships, and townships within supertownships. Their experimental model (Krumbein and Slack, 1956, p. 754) is given as:

$$
X_{i j k m}=\mu+\alpha_{i}+\beta_{i j}+\gamma_{i j k}+\delta_{i j k m},
$$

where $X_{i j k m}$ is the radioactivity of the $m$ th sample $(1 \leq m \leq d)$ from the $k$ th mine $(1 \leq k \leq c)$ in the $j$ th township $(1 \leq j \leq b)$ in the $i$ th supertownship $(1 \leq i \leq a)$. The term " $\mu$ " is the grand mean radioactivity for all potential samples in the shale bed (the true mean), $\mu+\alpha_{i}$ is the true mean for the $i$ th supertownship, $\mu+\alpha_{i}+\beta_{i j}$ is the true mean for the $j$ th township in the $i$ th supertownship, and $\mu+\alpha_{i}+\beta_{i j}+\gamma_{i j k}$ is the true mean for the $k$ th mine in the $j$ th township in the $i$ th supertownship. If a sampling design based on this model is used, the total sampling error is spread over several levels. As the purpose of each sample is to allow estimation of a mean for a mine (the quantity $\mu+\alpha_{i}+$ $\left.\beta_{i j}+\gamma_{i j k}\right)$, the mean of the $\delta_{i j k m}$ components $\left(\frac{1}{d} \sum_{m=1}^{d} \delta_{i j k m}\right)$ may be regarded as the mean sampling error associated with the mine. As the purpose in sampling mines is to allow estimation of a township mean $\left(\mu+\alpha_{i}+\beta_{i j}\right)$, the mean of the $\gamma_{i j k}$ and $\delta_{i j k m}$ components $\left(\frac{1}{c d} \sum_{k=1}^{c} \sum_{m=1}^{d} \gamma_{i j k}+\right.$ $\left.\delta_{i j \mathrm{~km}}\right)$ may be regarded as the mean sampling error for the township. Similarly, the mean of the $\beta_{i j,} \gamma_{i j k}$, and $\delta_{i j k m}$ components $\left(\frac{1}{b c d} \sum_{j=1}^{b} \sum_{k=1}^{c} \sum_{m=1}^{d} \beta_{i j}+\gamma_{i j k}+\delta_{i j k m}\right)$ is the mean sampling error for the supertownship. In Krumbein and Slack's experiment, all supertownships in the area of interest were sampled, so no error was involved in selection of supertownships-the master sampling units, or major sampling localities.

Overall bias, variable bias, and variable precision, discussed in the previous section, and defined in terms of the model in equation 4, are equally applicable to more specialized models such as that given in equation 11. These several kinds of error, however, will now occur at the several levels of sampling. For example, the selection of mines may involve an overall bias, but selection of townships may be unbiased yet cause variable precision from one supertownship to another.

The purpose here is to show how the concept of sampling error used in the previous section can be extended to more complex sampling plans. The concept of sampling error will be applicable whenever a collected specimen, or group of specimens, is intended to represent a sampling locality, the locality consisting of some part of the rock body larger than an individual specimen. 


\section{ANALYTICAL ERROR}

The preceding discussion has been based on the assumption that no analytical error is present in the experiment. In studies where the effects of analytical error are evaluated, the model must be extended to:

$$
X_{i j k}=\mu+\beta_{i}+\omega_{i j}+\alpha_{i j k},
$$

where $\alpha_{i j k}$ is the difference between the $k$ th analytical determination $\left(X_{i j k}\right)$ on the $j$ th specimen from the $i$ th locality and the correct concentration of the constituent in the specimen $\left(\mu+\beta_{i}+\omega_{i j}\right)$. The term " $\alpha_{i j k}$ " is the error associated with an individual analysis. The model in equation 12 is applicable in experiments designed to evaluate analytical error and to determine the variance of sampling error, $\omega_{i j}$, separate from that of the analytical error. The model may be extended further for experiments designed to separately evaluate parts of the analytical procedure or methods of sample preparation (such as crushing and sieving) (Shaw, 1961).

Like the special model described in the previous section, the model in equation 12 is useful in preliminary studies; here the studies are designed, in part, to determine whether an analytical method is sufficiently precise for a particular field problem. If the variance of $\alpha_{i j k}$ is not small in comparison to the variance of $\omega_{i j}$ or $\beta_{i}$, the method of analysis may be inadequate for describing variance among specimens within localities, or among localities within the rock body.

In final sampling programs we are more concerned with the mean error associated with a specimen (the error of the mean of all analyses on the specimen) than with the error of an individual analysis, and it will be convenient to designate this mean error by the symbol $\theta_{i j}$, where,

$$
\theta_{i j}=\frac{1}{p} \sum_{k=1}^{p} \alpha_{i j k}
$$

The quantity $\theta_{i j}$ is the difference between the true concentration in the specimen and the mean of the $p$ analyses of that specimen. The error in the mean analysis of a specimen is the important factor in field geochemical problems, even though the error in individual analyses determines the nature of the mean error. Of course, where only one analysis is made per specimen, $\theta_{i j}=\alpha_{i j 1}$.

If $\theta_{i j}$ is part of the model, the analytical error is placed on a level with sampling error, and, even though the model in equation 12 is used in evaluating analytical error, the role of the error in the type of geochemical field problems with which we are concerned here is best described in terms of equation 14:

$$
X_{i j}=\mu+\beta_{i}+\omega_{i j}+\theta_{i j} .
$$

In considering total experimental error-that due to both sampling and to analysis-it will be convenient to use:

or

$$
X_{i j}=\mu+\beta_{i}+(\omega+\theta)_{i j}
$$

$$
X_{i j}=\mu+\beta_{i}+\epsilon_{i j}
$$

where $\epsilon=\omega+\theta$, the total sampling and analytical error associated with a specimen. Actually, it is the component of total experimental error, $\epsilon_{i j}$, that is of major concern in data interpretation, even though the character of the total error is determined by the character and magnitude of sampling and analytical errors present. It is possible, and even likely in some studies, that either sampling or analytical error can be overwhelmingly dominant and thus control the nature of the total error. The advantages of high analytical precision, for example, might be almost lost where the sampling variance is large, or a small sampling bias may be insignificant compared with a large analytical bias. Similarly, skewed analytical error with small variance might be almost completely obscured by normally distributed sampling error with large variance; the deviation of the total error from a normal distribution might be undetectable and unimportant. However, characteristics of both the sampling errors and the analytical errors are reflected in the total error to some degree, and the individual components $\omega_{i j}$ and $\theta_{i j}$ in equation 15 are still of basic importance. It would be rare indeed for undesirable features of sampling and analytical errors to compensate in such a way that the features were not present in the total error. Unbiased normally distributed sampling and analytical errors, of variance $s_{\omega_{i}}^{2}$ and $s_{\theta_{i}}^{2}$, respectively, will give rise to unbiased normally distributed total error with variance $s_{\omega_{i}}^{2}+s_{\theta_{i}}^{2}$ (if the sampling and analytical errors are independent).

Determination of the absolute value of $\theta_{i j}$ for any set of analyses is not generally possible because the correct concentration of any constituent in a rock specimen is never known. The value of $\theta_{i f}$, or of $\alpha_{i j k}$, can be closely estimated for analyses of artificially prepared standards, however, and this is common laboratory practice in the evaluation of a new analytical technique. In spite of the fact that $\theta_{i j}$, the mean analytical error for a specimen, is never known in an actual field experiment, we are faced with the necessity of making judgments about this value prior to data interpretation, just as we must judge the character of the sampling error, $\omega_{i j}$.

Three aspects of analytical error, $\theta_{i j}$, as for sampling error, are important in efforts to detect and describe variation among sampling localities: overall bias, variable bias, and variable precision. Also, the frequency 
distribution of the analytical error may be important, especially when normal probability theory is employed in data interpretation.

Overall blas

Overall bias is absent when the mean analytical error for all specimens and all localities tends toward zero as the number of localities and specimens per locality is increased.

$$
\frac{1}{m n} \sum_{i=1}^{m} \sum_{j=1}^{n} \theta_{i j} \rightarrow 0 \text {, as } m, n \rightarrow \infty \text {. }
$$

If equation 17 is not true, overall analytical bias is present. This may result from a large number of causes (including sample contamination) that may occur at any time from when the sample was collected to when the analytical determination is received by the geologist. (This all-inclusive notion of analytical error is not fair to the analyst, but errors due to sample handling and preparation must be accounted for somewhere. The part of the analytical error that is the responsibility of the analyst may be referred to as laboratory error when such subdivision is necessary.)

There are numerous ways in which overall bias and other types of error could occur in analysis. Many are well known to chemists and other analysts, and examples could be given with reference to any particular analytical method. However, it may be useful here to refer to the well-known method of estimating uranium content by measurement of radioactivity. In the commonly used laboratory method a small powdered rock specimen is placed in a lead chamber containing radioactivity counters of various types; the lead chamber eliminates nearly all radioactivity other than that from the specimen itself. Results of the radioactivity measurements are commonly expressed in units of equivalent uranium (percentage eU), where equivalent uranium is defined as the ratio of the net counting rate of a specimen to the net counting rate per percentage of a uranium standard in equilibrium with all of its disintegration products, both measured under similar geometry (Rosholt, 1954, p. 1307). Viewed as a measurement of radioactivity, the eU analysis is generally unbiased. As a method of uranium analysis, its accuracy depends on two main assumptions:

1. That all radioactivity in the specimen is from uranium and its radioactive decay products.

2. That uranium and all its radioactive decay products are in equilibrium within the specimen; that is, each radioactive member of the uranium series is disintegrating within the specimen at the same rate at which it is being formed.

The first assumption may be invalid when, for example, part of the radioactivity from the specimen arises from thorium or potassium, rather than from the uranium series. The second assumption may be invalid when radioactive decay products of the uranium series have been removed from the specimen or where they are present in excessive amounts. Either situation can give rise to overall bias in uranium assays by the eU method. The mean of the assay error may tend toward some positive or negative value, rather than toward zero. Rosholt (1959) discussed this subject thoroughly.

Overall bias in low-level radiometric uranium determinations was illustrated by Newman (1962, table 33). The data consist of fluorimetric uranium and $\mathrm{eU}$ analyses of 95 mudstone specimens. The fluorimetric analyses indicate that most of the specimens contain less than 10 parts per million uranium; the radiometric analyses are somewhat higher and, if the fluorimetric analyses are accepted as correct, have an average error of plus 13 parts per million. The bias is probably due to the presence of potassium (containing $\mathrm{K}^{40}$ ) in the analyzed specimens (Newman, 1962, table 33 ).

variable bias

Variable bias is absent when the mean analytical error tends to be the same from one locality to another as the number of specimens per locality is increased. That is,

$\frac{1}{n_{1}} \sum_{j=1}^{n_{1}} \theta_{1 j} \rightarrow \frac{1}{n_{2}} \sum_{j=1}^{n_{2}} \theta_{2 j} \rightarrow \ldots \frac{1}{n_{i}} \sum_{j=1}^{n_{i}} \theta_{i j}$, as $n_{1}, n_{2}, \ldots n_{i} \rightarrow \infty$.

If equation 18 is not true, variable analytical bias is present. Analytical bias that is variable from one sampling locality to another may result when the specimens from different localities are treated differently or when the localities' characteristics that affect the analy tical procedure vary. For example, if the analytical work extends over a long period of time, improvements in technique or analysts are likely to occur. If the specimens are treated locality by locality rather than in randomized sequence, the analytical bias associated with localities can easily vary. Variation in the chemical or physical characteristics of sampling localities can also cause variable bias. Where the rock at some sampling localities contains large amounts of one constituent that interferes with the determination of another constituent, variable bias could occur if suitable precautions were not taken. In some spectrographic procedures, for example, high concentrations of manganese may interfere with the determination of silver (Bastron and others, 1960, p. 179). If steps had not been taken to allow for the interference, biased determinations of silver would result only on specimens from localities where manganese concentrations are high. 
Finnell, Franks, and Hubbard (1963, p. 46-49) discussed an excellent example of variable bias in eU analyses among sampling localities within uraniumcopper deposits in San Juan County, Utah. Because of differential uranium leaching, specimens from weathered parts of the outcrop contain less uranium than the eU analysis would indicate, whereas unweathered specimens from underground contain about the same amounts of uranium as indicated by the eU analyses. If the eU analyses had been viewed as uranium determinations rather than as measurements of radioactivity, the bias would have ranged from some high positive value at localities where weathered outcrop was present to some value near zero at unweathered localities.

Variable bias from one sampling locality to another may also result when the amount of bias is related to the amount of the constituent present. In some types of colorimetric methods an analyst may tend to overestimate low concentrations and underestimate high concentrations. As the amount of the constituent present can be expected to vary from one sampling locality to another, the bias related to the amount present also varies among localities. Bias related to the amount present may also result from incorrect standards or from the chemistry involved in the analysis itself.

Examples of both overall bias and bias that varies with amount of constituent present were given by Wayman and Miesch (1965), with regard to field methods for determining the amounts of detergent in water. Analyses of prepared standards containing 0-1 part per million detergent (alkylbenzenesulfonate) were made by two analysts using three methods. Some of the results are given graphically in figure 3. Example $A$ is from a standard laboratory technique; the mean of the deviations from the accepted values of the prepared standards is about zero at each level of concentration. Neither overall nor variable bias can be demonstrated. In example $B$, based on a rapid field method, the bias present is clearly related to concentration; it is small or absent at low and high concentrations but large (and negative) at intermediate concentrations. As concen trations at different sampling localities may certainly vary when the technique is applied in a field problem, variable bias among the localities is certain to result. As the biases are not compensating, an overall negative bias will also be present.

variable precision

Variable precision in analysis is absent when the variance of the analytical error associated with specimens, $\theta_{i j}$, tends to be the same from one sampling locality to another as the number of specimens per locality is increased. That is,

$$
s_{\theta_{1}}^{2} \rightarrow s_{\theta_{2}}^{2} \rightarrow \ldots s_{\theta_{i}}^{2} \text {, as } n_{1}, n_{2}, \ldots n_{i} \rightarrow \infty \text {. }
$$

If equation 19 is not true, variable analytical precision is present in the data. This type of error may be expected when the samples from various localities are treated differently or when characteristics of the localities that affect the analytical precision vary.

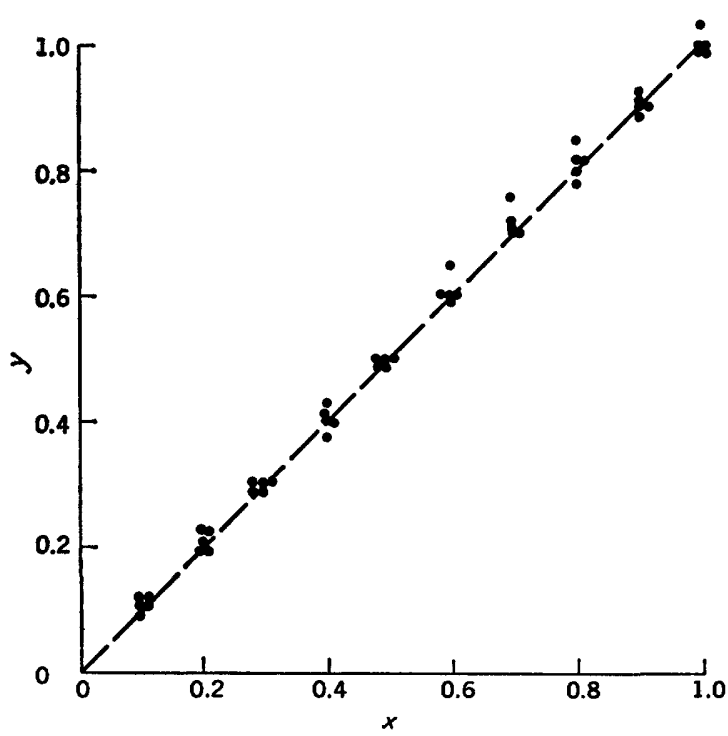

A. LABORATORY METHOD

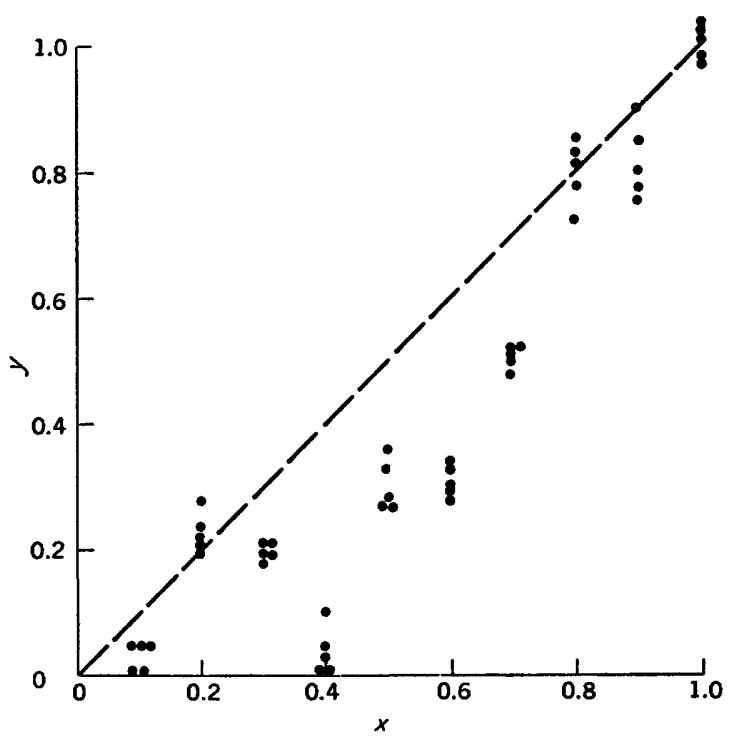

B. FIELD METHOD

Figure 3.-Analytical determinations on prepared standards of detergent in water. $X=$ concentration in standard; $Y=$ measured concentration. Concentrations are given in parts per million. From Wayman and Miesch (1965). 
The conditions under which variable precision is likely to occur are about the same as those that might lead to variable analytical bias. It seems, however, that variable analytical precision is much more common than variable bias, especially in studies directed at minor elements. The precision of minor-element determinations, as is well known, nearly always varies with the concentration. Fortunately, however, variable analytical precision is an easily recognized type of error and, because it is frequently systematic, can often be corrected by suitable data transformations (Bennett and Franklin, 1954, p. 356). Variable precision may also be corrected by making a number of replicate analyses of each sample proportional to their variance-by adjusting $p$ in equation 13 proportional to the variance of $\alpha_{i j k}$.

Variable precision of the analytical error, $\alpha_{i j k}$ in equation 12 , from one specimen to another may be detected by computing the variance of analy tical values, $X_{i j k}$, for each specimen and applying several statistical tests for variance homogeneity (see Bennett and Franklin, 1954, p. 196-200). The same tests may be used to detect variable precision of the total sampling and analytical error, $\epsilon_{i j}$ in equation 16, among sampling localities.

\section{Frequency distribution}

The components of analytical error, $\theta_{i j}$, should tend to have a normal distribution. The frequency distribution of the total experimental error for sampling localities, $\epsilon_{i j}$ in equation 16 , may be examined by constructing the frequency distribution of analytical values, $X_{i j}$, representing the locality. If the distribution departs widely from the normal, it can commonly be corrected by suitable data transformations (Bennett and Franklin, 1954, p. 91). The requirement of a normal distribution is necessary only when probability theory is to be employed in data interpretation, al though symmetrical frequency distributions are easier to interpret by' any method.

The frequency distribution of the analytical error alone is best determined from a large number of replicate analyses of the same specimen, or from a large number of estimates of $\alpha_{i j k}$ obtained by comparing analytical values with corresponding values known to be more correct.

\section{EFFECTS OF ERROR TYPES IN STATISTICAL ANALYSIS}

In the preceding discussion several types of sampling and analy tical error have been described, and the effects of each error type in data interpretation are briefly discussed. The effects are the same whether the error is due to sampling or to analysis.

Overall bias is present when the mean of the error components does not tend toward zero, and its effect is merely to distort the estimate of the mean composition of the rock body. Unless the bias is variable from one sampling locality to another, the estimated locality means tend to be wrong by a constant amount, and estimates of differences among locality means tend to be correct.

Bias that is variable from one sampling locality to another may have disastrous effects on data interpretation. As the bias for each locality varies and is unknown, determining the differences among localitiesthe primary objective of most geochemical field sampling - may be impossible.

Variable precision of sampling and analysis from one locality to another presents difficulties in judging both the statistical and geologic significance of differences but does not present as severe difficulties as does variable bias.

The type of frequency distribution of errors is of even less concern in data interpretation, though symmetrically distributed error is easier to evaluate by both formal statistical or other methods. When some types of probability theory are used in data interpretation, however, the type of frequency distribution displayed by the error components should be considered.

In formal statistical methods of data analysis, certain assumptions pertaining to the kinds of sampling and analytical error are required to make valid use of statistical models as given in previous parts of this paper. These models are the bases for formal statistical analysis of the data. However, it is a reasonable circumstance that the required assumptions regarding data errors are much the same for formal statistical analysis as for other methods of data analysis. Moreover, the relative importance of error assumptions required for formal statistical methods is about the same as for other methods. The lone exception here is the importance of the normal distribution in statistical methods based on normal probability theory.

The required assumptions regarding data errors are about the same for all statistical methods, even though the requirements of the data themselves, and the manner in which the data are collected, may differ. Multivariate statistical methods involve some assumptions regarding data errors, such as uncorrelated errors among variables, that are not considered here; but the requirements of most statistical methods that deal with one estimated variable at a time can be illustrated by reviewing two methods that have been shown to be useful in geochemical problems. These methods are analysis of variance and trend-surface analysis (polynomial multiple regression). Applications of these methods to geochemical and petrologic problems are so numerous that citation of individual papers is unnecessary. 


\section{ANALYSIS OF VARIANCE}

Analysis of variance has been applied in geochemical problems for testing compositional differences for statistical significance and for partitioning the total variability of a constituent among several factors. Applications of the second type are efforts to ascribe variability to geologic or spatial factors in the correct proportions. The assumptions that are required for analysis of variance techniques in general were summarized by Eisenhart (1947). These assumptions, as stated for the general case, appear somewhat formidable; viewed in terms of a particular problem, they are more easily understood. In a companion paper to Eisenhart's, Cochran (1947) reviewed the effects of failure of the data to meet the requirements set forth by Eisenhart. Insofar as experimental error is concerned, Cochran assumed that all the errors have zero means, and he tabulated the other assumptions required as follows:

1. Errors must be independent.

2. Errors must have a common variance.

3. Errors should be normally distributed.

If the assumption of zero means among the errors fails (overall bias is present), the analysis of variance may still be valid so long as the errors are independent, have a common variance, and are normally distributed. The grand mean and individual means for sampling localities will be biased, but the analysis of variance method is still valid for testing the significance of differences among them.

The three error requirements will be discussed as applicable to analysis of variance based on the model given in equation 16.

Errors lack independence when the amount of one error is related to that of another. This may occur in geochemical field sampling when the errors associated with specimens from one locality tend to be distributed about one value and those associated with specimens from another locality tend to be distributed about a different value. The errors, then, are related to the localities and lack independence. The errors associated with at least one of the localities, moreover, are biased, and the bias is variable among localities. The effect of variable bias, or dependent errors, in analysis of variance is that the additivity of variances is destroyed, as shown below in an example analogous to one given previously:

$\begin{array}{ccccc}i & j & X_{i j} & =\left(\mu+\beta_{i}\right)+ & \epsilon_{i j} \\ 1 & 1 & 1 & 2 & -1 \\ 1 & 2 & 4 & 2 & +2 \\ 2 & 1 & 6 & 4 & +2 \\ 2 & 2 & 1 & 4 & -3\end{array}$

Here the mean of the $\epsilon_{i j}$ values $(-1$ and +2$)$ added to $\mu+\beta_{i}=2$ is different from that of the $\epsilon_{i j}$ values $(+2$ and -3$)$ added to $\mu+\beta_{i}=4$. The variance of $\mu+\beta_{i}(=1,3)$ plus the variance of $\epsilon_{i j}(=6)$ is equal to $7 \frac{1}{3}$, whereas that of $X_{i j}$ is 6 . Because the additive character of variance is destroyed by variable bias, the analysis of variance method, based on models given in this paper, is invalid for estimating variances components and testing the significance of differences among sampling locality means. Mean square esimates derived in the analysis of variance procedure tend to be incorrect.

Common variance among the errors is absent when the errors in one category of the experimental design have a different variance than those in another category. This occurs in geochemical field problems when the precision of sampling or analysis, measured by the variance, varies from one sampling locality to another. Variable precision prohibits valid estimation of mean squares in analysis of variance because the true component of error variance is not a single value, but a range of values. Data transformations that may be suitable for obtaining a common, or homogeneous, variance were reviewed by Bartlett (1947).

The requirement of a normal distribution for experimental errors does not affect the validity of the analysis of variance method for estimation of variance components, but it does affect tests for significance of differences among sampling locality means. The effects, however, are not severe, and non-normality is not regarded as a rigid requirement when $F$-tests are applied (Cochran, 1947, p. 24). The $F$-tests, because of this lack of high dependence on a normal distribution, are said to be "robust." When the normal distribution assumption cannot be satisfied, nonparametric methods of statistical analysis may be useful (Siegel, 1956).

Two-tailed $t$-tests for estimating the significance of difference between two means-a special application of analysis of variance-and methods for estimating the confidence interval of a mean based on the $t$ distribution are also "robust"; the normal-distribution requirement is not severe. On the other hand, methods based on the one-tailed $t$-distribution, as for comparing a mean with some critical value, lack "robustness," and the normal-distribution requirement is more critical (Cochran, 1947, p. 24-25). The lack of "robustness" of Bartlett's test for common, or homogeneous, variance is well known (Box, 1953).

\section{TREND ANACYSIS}

The method of trend analysis, as currently applied to geologic problems, is a form of multiple regression wherein polynomial or other mathematical surfaces are fitted to map data by least-squares techniques. Several 
objectives can be achieved by this technique, but chief among these is the separation of total map variability into regional and local components. The essential features of the method, as applied in geology, were summarized by Krumbein (1959). Trend analysis may be viewed as an empirical tool or as a descriptive technique in geologic data analysis; as such, the data errors involved are not subject to unusual requirements. Overall bias in the data merely affects the estimation of the constant term in the computed regression equation and the absolute values on the contours of the regression surface. Variable bias affects the regression coefficients and the form of the fitted surface. Variable precision results in unequal reliability of the locality means described by the fitted surface. These effects are the same as would be present if the data were merely contoured. The frequency distribution of the error has no unique effect on either trend analysis or contouring procedures.

Testing the statistical significance of fitted trend surfaces, by $F$-tests as outlined in Bennett and Franklin (1954, p. 431-436), requires the same assumptions regarding data errors as listed previously. Other assumptions regarding the data and the mathematical function fitted to the data in trend analysis are beyond the scope of this paper. (See Link and others, 1964.)

From the above examples it is apparent that data containing errors causing the data to be unsuitable for statistical analysis are probably unsuitable for any other rigid type of interpretation. When neither overall bias, variable bias, nor variable precision of the errors is of large magnitude, the data, insofar as the error is concerned, are suitable for interpretation on either statistical or substantive grounds, although statistical methods are more likely to overcome the effects of unbiased error. However, statistical methods have no greater power to overcome effects of overall and variable bias than have other methods.

\section{SUMMARY}

The entire purpose of statistical analysis in geochemical field problems is to evaluate and allow for the effects of error in the data in drawing geochemical conclusions. However, neither statistical nor other types of data analysis can be effective when certain kinds or error are present. Some kinds of error can be effectively reduced by collecting more data or by making data transformations; but other kinds, if of sufficient magnitude, may persist and invalidate any type of data interpretation.

The individual components of sampling and analytical error are never known in a real field problem, though the variance of total experimental error and the form of its frequency distribution can be determined. In spite of the fact that the error components are unknown, we are faced with the necessity of making judgments about them prior to any sort of data interpretation. These judgments are necessarily based on knowledge of the sampling and analytical procedures and the circumstances under which they were carried out. No sampling or analytical procedure gives results that are perfectly precise, or reproducible; some imprecision is present in all observational data. Overall bias, variable bias, and variable precision, however, may or may not be present in important amounts, and the presence of one is not necessarily related to the presence of another. In some studies the occurrence of these error types can be reduced or controlled by suitable precautions in the field and laboratory. In other studies, owing to the nature of the rock body and its outcrop characteristics or the nature of an analytical method that must be used, the occurrence of these error types in important amounts is beyond control.

Overall bias may be controlled when it is due to misjudgment on the part of the sampler or analyst. The sampler may be persuaded to use an objective sampling technique, and the analyst may change his analytical methods. Overall bias may also result, however, under circumstances beyond the sampler's or analyst's control, as when the sampler is restricted to outcropping parts of the rock body and the outcrop is not representative of the rock body as a whole. Overall bias in analysis may be beyond control, for practical purposes, when a method that must be used, owing to cost or other factors, involves such shortcomings as inadequate sample digestion or inadequate complexing or precipitation. Overall bias in analysis is subject to tests by analysis of prepared standards or by comparison with other methods accepted as more precise and accurate, but bias in sampling can only be inferred from knowledge of the field procedures and the nature of the rock body.

Variable bias in sampling and analysis, where it is due to variation in the field and laboratory procedures, may be reduced by exercising precautions to keep the procedures uniform. However, variable bias may be caused by circumstances that are beyond control, as when outcrop characteristics differ from one sampling locality to another and when attributes of the rock that affect analytical bias vary among localities. Variable sampling bias may only be inferred from knowledge of the field conditions and procedures, but variable analytical bias sometimes may be detected by analysis of prepared standards or by comparison with methods known to be more precise and accurate.

Variable precision in sampling, like overall and variable sampling bias, will rarely be introduced by the sampler who uses some objective method of sample 
selection. Variable precision resulting, however, from natural differences in the amount of local variation over different parts of the rock body is unavoidable. Variable sampling precision is also unavoidable when caused by the variable nature of the outcrop from one sampling locality to another, if only outcrops can be sampled. Analytical precision that varies with the amount of the constituent present is an inherent feature of most analytical methods for minor-element determinations and is generally unavoidable. Variable precision in total experimental error, that due to both sampling and analysis, is comparatively easy to detect in the analytical data.

Experimental results which are affected by bias in sampling cannot be corrected; effects of analytical bias may be corrected in certain restricted instances where it is either constant or systematically variable (Youden, 1962). The effects of variable precision commonly may be overcome by suitable data transformations; these same transformations may also bring the frequency distribution closer to normal (Bartlett, 1947, p. 40). Variable precision among sampling locality means may be overcome by collecting additional specimens at the more variable localities.

The effects of overall bias in geochemical errors are usually not severe, unless the purpose of the investigation is to estimate absolute mean concentrations of constituents to several significant figures. Variable bias, however, may seriously affect both statistical or intuitive interpretation of the data. Variable precision or a non-normal frequency distribution of error components renders judgments of the relative significance of differences difficult and may prevent use of some useful statistical methods based on probability theory.

Despite the difficulties that can and do arise in the field and in the laboratory, sound geological judgment in designing a study and use of objective sampling plans can do much to reduce their effects. The many valid inferences made in geochemical studies attest to the fact that in many instances the errors are not great enough to invalidate the findings of the study. Nevertheless, we cannot afford to igmore the implications of error. The different types of error that may be present must be recognized and the kinds of effects each may have on data interpretation should be known. Only through a clear understanding of these factors can we arrive at sampling designs and methods of data interpretation that are both consistent with the geologic problem under consideration and efficient with respect to the available field and laboratory resources.

\section{LITERATURE CITED}

Baird, A. D., MeIntyre, D. B., Welday, E. E., and Madlem, K. W., 1964, Chemical variations in a granitic pluton and its surrounding rocks: Science, v. 146, no. 3641, p. 258-259.

Bartlett, M. S., 1947, The use of transformations: Biometrics, v. 3 , no. 1 , p. $39-52$.

Bastron, Harry, Barnett, P. R., and Murata, K. J., 1960, Method for the quantitative spectrochemical analysis of rocks, minerals, ores, and other materials by a powder $\mathrm{D}-\mathrm{C}$ arc technique: U.S. Geol. Survey Bull. 1084-G, p. 165-182.

Bennett, C. A., and Franklin, N. L., 1954, Statistical analysis in chemistry and the chemical industry: New York, John Wiley \& Sons, $724 \mathrm{p}$.

Box, G. E. P., 1953, Non-normality and tests on variances: Biometrika, v. 40, p. 318-335.

Cochran, W. G., 1947, Some consequences when the assumptions for the analysis of variance are not satisfied: Biometrics, v. 3 , no. 1 , p. 22-38.

Cochran, W. G., Mosteller, F., and Tukey, J. W., 1954, Principles of sampling: Am. Statistical Assoc. Jour., v. 49, p. 13-35.

Eisenhart, Churchill, 1947, The assumptions underlying the analysis of variance: Biometrics, v. 3, no. 1, p. 1-21.

Finnell, T. L., Franks, P. C., and Hubbard, H. A., 1963, Geology, ore deposits, and exploratory drilling in the Deer Flat area, White Canyon district, San Juan County, Utah: U.S. Geol. Survey Bull. 1132, 114 p.

Krige, D. G., 1960, On the departure of ore value distributions from the lognormal model in South African gold mines: South African Inst. Mining and Metallurgy Jour., v. 61, no. 4, p. 231-244.

Krumbein, W. C., 1959, Trend surface analysis of contour-type maps with irregular control-point spacings: Jour. Geophys. Research, v. 64, no. 7, p. 823-834.

- 1960, The "geological population" as a framework for analysing numerical data in geology: Liverpool and Manchester Geol. Jour., v. 2, pt. 3, p. 341-368.

Krumbein, W. C., and Slack, H. A., 1956, Statistical analysis of low-level radioactivity of Pennsylvania black fissile shale in Illinois: Geol. Soc. America Bull., v. 67, no. 6, p. 739-762.

Laffitte, Pierre, 1957, Introduction a l'étude des roches métamorphiques et des gites métalliféres: Paris, Masson et Compagnie, 343 p.

Link, R. F., Koch, G. S., Jr., and Gladfelter, G. W., 1964, Computer methods of fitting surfaces to assay and other data by regression analysis: U.S. Bur. Mines Rept. Inv. 6508, $69 \mathrm{p}$.

Miesch, A. T., 1964, Effects of sampling and analytical error in geochemical prospecting, in Parks, G. A., ed., Computers in the mineral industries, pt. 1: Stanford Univ. Pubs. Geol. Sci., v. 9, no. 1, p. 156-170.

Miesch, A. T., and Connor, J. J., 1964, Investigation of samplingerror effects in geochemical prospecting, in Short papers in geology and hydrology: U.S. Geol. Survey Prof. Paper 475-D, p. D84-D88.

Newman, W. L., 1962, Distribution of elements in sedimentary rocks of the Colorado Plateau-A preliminary report: U.S. Geol. Survey Bull. 1107-E, p. 337-445.

Ostle, Bernard, 1963, Statistics in research: 2d ed.. Ames, Iowa, Iowa State College Press, 585 p. 
Rosholt, J. N., 1954, Quantitative radiochemical method for determination of major sources of natural radioactivity in ores and minerals: Anal. Chemistry, v. 26, no. 8, p. 13071311.

1959, Natural radioactive disequilibrium of the uranium series: U.S. Geol. Survey Bull. 1084-A, p. 1-30.

Shaw, D. M., 1961, Manjpulation errors in geochemistry: A preliminary study: Royal Soc. Canada Trans., v. 55, ser. 3, sec. 4, p. 41-55.

Shoemaker, E. M., Miesch, A. T., Newman, W. L., and Riley, L. B., 1959, Elemental composition of the sandstone-type deposits, in Garrels, R. M., and Larsen, E. S., 3d, compilers, Geochemistry and mineralogy of the Colorado Plateau uranium ores: U.S. Geol. Survey Prof. Paper 320, p. 25-54.
Sichel, H. S., 1952, New methods in the statistical evaluation of mine sampling data: London, Inst. Mining Metallurgy Trans., v. 61, p. 261-288.

Siegel, Sidney, 1956, Nonparametric statistics for the behavioral sciences: New York, McGraw-Hill Book Co., 312 p.

Tippett, L. H. C., 1952, The methods of statistics: 4th ed., New York, Dover Pub. Inc., 395 p.

Wayman, C. H., and Miesch, A. T., 1965, Accuracy and precision of laboratory and field methods for the determination of detergents in water: Water Resources Research, v. 1, no. 4 , p. 471-476.

Youden, W. H., 1962, Accuracy of analytical procedures: Assoc. Official Agr. Chemists Jour., v. 45, no. 1, p. 169-173. 